\title{
Observability in dynamic evolutionary models
}

\author{
López, I.* Gámez, M. and Carreño, R. \\ Departamento de Estadística y Matemática Aplicada,Universidad de Almería \\ La Cañada de San Urbano sn 04120-Almeria (SPAIN)
}

\begin{abstract}
In the paper observability problems are considered in basic dynamic evolutionary models for sexual and asexual populations. Observability means that from the (partial) knowledge of certain phenotypic characteristics the whole evolutionary process can be uniquely recovered. Sufficient conditions are given to guarantee observability for both sexual and asexual populations near an evolutionarily stable state.
\end{abstract}

Key words: evolutionary game, replicator dynamics, strategic evolutionary model, observability of nonlinear systems

\section{Introduction}

The static concept of an evolutionarily stable state (ESS) was introduced by Maynard Smith and Price (1973) to describe the terminal state of phenotypic evolution of an asexual population. ESS is a state of the population in which no rare mutant phenotype can propagate via natural selection. (A formal definition of ESS in terms of the pay-off matrix of the evolutionary game is recalled in section 3.)

Taylor and Jonker (1978) proposed a dynamic evolutionary model (evolutionary game dynamics, replicator dynamics) and proved (see also Zeeman

${ }^{*}$ Corresponding author: I. López, fax:(34)950-015167, email: milopez@ual.es

Running head: Observability in dynamics evolutionary models. 
(1979), Hofbauer and Sigmund (1988)) that ESS is an asymptotically stable equilibrium for this dynamics.

Later, the notion of evolutionary stability was extended to sexual populations defining the corresponding dynamic model, the so-called strategic model of viability selection, where in a diploid model the state of the population is described in terms of allele frequencies, and at the phenotypic level an evolutionary game models the selection process. (See Creesman et al. 1996, Garay and Varga 1998, Cressman et al. 2003).

In the above dynamic models, an important qualitative property, asymptotic stability (in particular the convergence to the evolutionarily stable state) was proved. Further important qualitative properties of these models can be explored applying concepts and methods of mathematical systems theory. This discipline was developed to deal with problems of system engineering, see Kalman et al. (1969). Its application to dynamic selection models was initiated by Varga (1989). In his paper controllability of Fisher's selection model was considered. In biological terms the main result was the following: If in a population subject to natural selection, certain perturbation changes the state of the population from a polymorphic equilibrium to a nearby state, then under generic conditions on the fitness matrix the population can be controlled by artificial selection into this equilibrium in given time. Recently, in Scarelli and Varga (2002) these results have been extended to selectionmutation processes.

In Varga (1992) selection processes were studied from another aspect of mathematical systems theory, namely, observability of Fisher's selection model was considered. A strong biological motivation for observability in population genetic models is the following: Frequency-dependent selection models are usually described in terms of allele frequencies, the macroscopic observation of the population, however, is carried out in terms of phenotypic characteristics. As a matter of fact, the dominance structure among alleles can obscure the underlying genetic process. In a static situation, in terms of the hereditary system, Garay and Garay (1998) gave a necessary 
and sufficient condition for the allele frequency-phenotype correspondence to be one-to-one. For the dynamic situation, in the strategic model of viability selection with symmetric genotype-phenotype correspondence (see Cressman et al. 1996, Garay and Varga 1998), the observability problem was investigated in Gamez et al. (2002) providing sufficient conditions to recover the genetic process observing only phenotypic characteristics.

In the present paper we extend these models to non-symmetric genotypephenotype correspondences, moreover, we also consider the observability problem in the classical replicator dynamics for the phenotypic evolution of an asexual population, in case of partial observation phenotype frequencies, providing sufficient conditions for observability. We notice that for evolutionary game dynamics controllability problems were considered in Kósa and Varga (1996), while the problem of partial observation in density-dependent models of population ecology was studied in Varga et al. (2002).

\section{Observability of nonlinear systems with in- variant manifold}

In this section we will recall the basic concepts and the sufficient condition given by Varga (1998) with additional comments on that condition.

Definition 2.1. For given $k \in\{1,2, \ldots, n-1\}$ a set $M \subset \mathbb{R}^{n}$ is called a regular $k$-dimensional sub-manifold, if there exists an open set $G \subset \mathbb{R}^{n}$ and a function $\phi \in C^{1}\left(G, \mathbb{R}^{n-k}\right)$ such that, for all $x \in G$, we have $R_{\phi^{\prime}(x)}=R^{n-k}$ and $M=\phi^{-1}(0)$.

For a continuously differentiable function $f: \mathbb{R}^{n} \rightarrow \mathbb{R}^{n}$, we consider the differential equation

$$
\dot{x}=f \circ x,
$$

where $\circ$ denotes the composition of functions (in traditional form $\dot{x}=f(x)$ ).

Given a regular $\epsilon$-dimensional sub-manifold $M \subset \mathbb{R}^{n}$, let $x^{*} \in M$ be such that $f\left(x^{*}\right)=0$ (in other words, an equilibrium of system (1)), then 
there exists a neighbourhood of $x^{*}$ and $T \in \mathbb{R}^{+}$such that any solution of (1) beginning at a point of this neighbourhood is defined in $[0, T]$.

For this section we suppose that $M$ is locally positively invariant for system (1) at $x^{*}$, that is, any solution of (1) beginning at a point of a neighbourhood of $x^{*}$ in $M$, remain in $M$.

For a given $m \in \mathbb{N}$, let $h: \mathbb{R}^{n} \rightarrow \mathbb{R}^{m}$ be a continuously differentiable function $h\left(x^{*}\right)=0$, we define an observation system as

$$
\left\{\begin{array}{l}
\dot{x}=f \circ x \\
y=h \circ x
\end{array}\right.
$$

Definition 2.2. We shall say that observation system (2) is locally observable (in $M$ ) at $x^{*}$; if there exists $\varepsilon \in \mathbb{R}^{+}$satisfying the following conditions:

Given $z^{i} \in M$, with $\left|z^{i}-x^{*}\right|<\varepsilon \quad(i \in\{1,2\})$ and

$$
\begin{aligned}
\dot{x}^{i}(t) & =f\left(x^{i}(t)\right) \quad(t \in[0, T]) \\
x^{i}(0) & =z^{i} \quad(i \in\{1,2\}) \\
h\left(x^{1}(t)\right) & =h\left(x^{2}(t)\right) \quad(t \in[0, T]) ;
\end{aligned}
$$

then $z^{1}=z^{2}$ (consequently $\left.x^{1}(t)=x^{2}(t) \quad(t \in[0, T])\right)$.

The local observability in $M$, at $x^{*}$ means that if, instead of the solution we can observe a transformation of it then, from this observed function we can recover the solution in a unique way, provided the solution begins at a point of $M$ near the given equilibrium $x^{*}$.

To formulate a sufficient condition which guarantees the local observability of system (2), we linearize system (2) at the equilibrium,

$$
L:=f^{\prime}\left(x^{*}\right), \quad C:=h^{\prime}\left(x^{*}\right),
$$

and define

$$
Q:=\left(\begin{array}{c}
C \\
C L \\
\cdot \\
\cdot \\
\cdot \\
C L^{n-1}
\end{array}\right)
$$


Now we recall a basic theorem proved by Varga (1989), in which a sufficient condition is given for local observability in "geometric" terms.

Theorem 2.3. Suppose that

$$
T_{*} \cap \operatorname{Ker} Q=\{0\} .
$$

Then system (2) is locally observable, where $T_{*}$ is the tangent space to $M$ at $x^{*}$.

Remark 2.4. For an insight to the transversality condition (3) let us consider a linear observation system

$$
\begin{aligned}
& \dot{x}=L x \\
& y=C x,
\end{aligned}
$$

without any condition on the invariance of a linear subspace. (Formally, with the notation of Definition 2.1 we would have $k=n, M=\mathbb{R}^{n}$ ). Then the classical necessary and sufficient condition for ("global") observability of the system is

$$
\operatorname{rank} Q=n,
$$

see e.g. Kalman (1969).

Since now $T_{*}=\mathbb{R}^{n}$, the condition of transversality is equivalent to the condition of Kalman (6). Let $K \subset \mathbb{R}^{n}$ be a $k$-dimensional linear subspace and suppose $K$ is positively invariant for system (4). According to condition (6) it would seem reasonable to think that for the ("global") observability inside the invariant subspace $K$ a sufficient condition would be

$$
\operatorname{rank} Q \geq k \text {. }
$$

As a matter of fact, this is not the case, as we can see in

Example 2.5. In space $\mathbb{R}^{2}$ let us consider the observation system

$$
\begin{aligned}
& \dot{x}=x \\
& y=C x \text { with } C:=[1,-1] .
\end{aligned}
$$


Then $K:=\left\{z \in R^{2} \mid z_{1}=z_{2}\right\}$ is a lineal subspace, positively invariant for the system. Now

$$
\operatorname{rank}\left[\begin{array}{c}
C \\
C L
\end{array}\right]=\left[\begin{array}{cc}
1 & -1 \\
1 & -1
\end{array}\right]=1=\operatorname{dim} K
$$

while the system is not observable because, for instance, both to the solution beginning at point $\left[\begin{array}{l}1 \\ 1\end{array}\right]$ and to the trivial solution there correspond the observation zero. (We can see that in this example we have $T_{*} \cap \operatorname{Ker} Q=$ $K \cap K \neq\{0\}$.) This situation explains why the condition of transversality and not the rank condition is the one that can be generalized to non-linear systems with invariant manifold.

Remark 2.6. For $n \in \mathbb{N}$ let us consider the standard simplex

$$
\Delta_{n}:=\left\{z \in \mathbb{R}^{n} \mid z \geq 0, \sum_{i=1}^{n} z_{i}=1\right\}
$$

It is easy to see that the interior $\stackrel{\circ}{\Delta}_{n}$ of this simplex is an $(n-1)$-dimensional sub-manifold in $\mathbb{R}^{n}$. In the following sections this will be the invariant manifold for the considered dynamics.

\section{Observability of the replicator model}

Now, let us recall the replicator model of phenotypic evolution of an asexual population. Given an $N \times N$ pay-off matrix $A$, we suppose that the individuals are replicators in the sense of having always the phenotype of the "father", this dynamics is given by the following model

$$
\dot{s}_{i}=s_{i}\left[(A s)_{i}-s A s\right] \quad(i \in 1,2, \ldots, N),
$$

where $s_{i}$ represents the frequency of individuals of type $i$ in the population and $(A s)_{i}=e_{i} A s$ is the mathematical expectation of the pay-off to an individual of $e_{i}$ type in terms of fitness, from its conflict with a randomly chosen 
individual of the population (see Hofbauer and Sigmund, 1988), while $s A s$ is the average phenotype of the population (here $e_{i}$ represent the $i$-th canonical basic vector of $\mathbb{R}^{N}$ ).

For an appropriate choice of an equilibrium $s^{*}$ we start out from the concept of ESS.

Definition 3.1.. A phenotypic state $S^{*} \in \Delta_{N}$ is called an evolutionarily stable strategy (ESS) if the following conditions hold:

i) equilibrium condition:

$s A S^{*} \leq S^{*} A S^{*}$ for all $s \in \Delta_{N}$,

ii) stability condition:

$s A s<S^{*} A s$ for all $s \in \Delta_{N} \backslash\left\{S^{*}\right\} \quad$ with equality in i)

It is easy to see that the set $\stackrel{\circ}{\Delta}_{N}$ with $C_{\phi}=\stackrel{\circ}{\mathbb{R}}_{+}^{N}$ and $\phi\left(x_{1}, \ldots, x_{N}\right)=$ $1-\sum_{i=1}^{N} x_{i}$ is an $(N-1)$-dimensional regular sub-manifold of $\mathbb{R}^{N}$.

We prove now that the simplex $\Delta_{N}$ and its interior $\stackrel{\circ}{\Delta}_{N}$ are positively invariant for replicator dynamics. Since these statements are often used in the literature withouth a proof or reference, for the reader's convenience we below provide the proofs. The invariance will be proved for a more general dynamics which can be also applied to the dynamics of section 4 . We shall consider the following dynamics:

$$
\dot{x}_{i}=x_{i}\left[g_{i}(x)-\sum_{j=1}^{N} x_{j} g_{j}(x)\right], \quad i \in\{1,2, \ldots, N\}
$$

where $g_{i}: \mathbb{R}^{N} \rightarrow \mathbb{R}$ are continuously differentiable functions. In our particular case

$$
g_{i}(x):=\sum_{j} a_{i j} x_{j}, \quad i \in\{1,2, \ldots, N\}
$$

where $a_{i j}$ are the entries of the corresponding pay-off matrix, and system (8) takes the form

$$
\dot{x}_{i}=x_{i}\left(\sum_{j} a_{i j} x_{j}-\sum_{l, r} a_{l r} x_{l} x_{r}\right), \quad i \in\{1,2, \ldots, N\}
$$


which is the classical replicator dynamics.

Theorem 3.2. The simplex $\Delta_{N}$ is positively invariant for the dynamics (8).

Proof. If we add the equations of system (8) we have

$$
\sum_{i} \dot{x}_{i}=\sum_{i} x_{i} g_{i}(x)-\sum_{i} x_{i} \sum_{j} x_{j} g_{j}(x) .
$$

Introducing the functions $\sigma:=\sum_{i} x_{i} ; b:=\sum_{i} x_{i} g_{i}(x)$, the equation (9) can be written as

$$
\dot{\sigma}=b-\sigma b=b(1-\sigma) .
$$

Then for the function $v=1-\sigma$ we obtain the equation

$$
\dot{v}(t)=-\dot{\sigma}(t)=-b(t) v(t), \quad\left(t \in \mathbb{R}_{+}\right) .
$$

Let $x(0) \in \Delta_{N}$ we have to prove $x(t) \in \Delta_{N}\left(t \in \mathbb{R}_{+}\right)$. To this end we verify the conditions:

A1) $\sum_{i} x_{i}(t)=1$.

A2) $x_{i}(t) \geq 0, \quad i \in\{1,2, \ldots, N\}$.

From $\sum_{i} x_{i}(0)=1$ we have $v(0)=0$ and therefore we the have the initial value problem

$$
\dot{v}(t)=-b(t) v(t) ; \quad v(0)=0,
$$

for a linear homogeneous differential equation. Its unique solution is $v \equiv 0$, that is, $\sigma(t)=\sum_{i} x_{i}(t)=1$ which proves $\left.A 1\right)$.

In order to see $A 2)$, fix $x(0) \in \Delta_{N}$.

Case 1. If $x_{i}(0)>0$ for all $i \in\{1,2, \ldots, N\}$ then also $x_{i}(t)>0$. Suppose the contrary: there exists some $i \in\{1,2, \ldots, N\}$ and $t_{1} \in \mathbb{R}$ such that $x_{i}(t)>0$ for all $t \in\left[0, t_{1}\right.$ [ and $x_{i}\left(t_{1}\right)=0$. Since $x_{i}$ is a solution of the $i$-th equation of (8), by the uniqueness of the solution, we have $x_{i} \equiv 0$ which is a contradiction to the condition $x_{i}(t)>0$ in $\left[0, t_{1}[\right.$.

Case 2. If $x_{i}(0)=0$ for some $i \in\{1,2, \ldots, N\}$, then according to what we have seen in the Case $1, x_{i}(t) \equiv 0$ for all $t$.

Therefore, joining both possibilities we have that $x(t) \geq 0$ for all $t$ which completes the proof. 
Remark 3.3. From the proof of this result we can also see that any subsimplex of $\Delta_{N}$ is positively invariant.

Theorem 3.4. The interior of simplex $\Delta_{N}$ is positively invariant for the $d y$ namics (8). In particular it is locally positively invariant at any equilibrium.

Proof. Let $x(0) \in \stackrel{\circ}{\Delta}_{N}$ Then from part $A 1$ ) of the proof of Theorem 3.2, we have that

$$
\sum_{i} x_{i}(t)=1, \quad\left(t \in \mathbb{R}_{+}\right)
$$

and from the Case 1 of the above proof it is verified that $x_{i}(t)>0$ for all $i \in\{1,2, \ldots, N\}$ and $t \in \mathbb{R}_{+}$, which completes the proof.

We present now results for $N=3$ in which sufficient conditions are provided under which the replicator dynamics model is locally observable at the equilibrium $s^{*}$ The latter means that, observing some of the phenotype frequencies we can recover the frequencies of the rest of phenotypes, provided that initially the vector of phenotypes was near $s^{*}$.

Theorem 3.5. Given a pay-off matrix $A \in \mathbb{R}^{3 \times 3}$. Let $s \in \stackrel{\circ}{ }_{3}$ an equilibrium and suppose

$$
-a_{12}+a_{13}+\sum_{i=1}^{3} s_{i}^{*}\left(a_{i 2}+a_{i 3}\right) \neq 0 .
$$

Then the observation system

$$
\left\{\begin{aligned}
\dot{s}_{i} & =f(s)=s_{i}\left[(A s)_{i}-s A s\right] \\
y & =h(s)=s_{1}
\end{aligned}\right.
$$

is locally observable in $\stackrel{\circ}{\Delta^{3}}$ at the equilibrium $s^{*}$.

Proof. Applying Theorem 2.3, let $z \in T_{*} \cap \operatorname{Ker} Q$ be, since $C=h^{\prime}\left(s^{*}\right)$, we have $C=\left(\begin{array}{lll}1 & 0 & 0\end{array}\right)$. On the other hand, from $z \in \operatorname{Ker} Q$ and $Q=\left(C, C L, C L^{2}\right)$ we obtain that $C z=0$. Hence we have

$$
z_{1}=0 \text { and } z_{2}=-z_{3}
$$


since $z \in T_{*}$ (that is, $z_{1}+z_{2}+z_{3}=0$ ).

Following Cressman (1996) we obtain that the linearization of equation (7) at the equilibrium $s^{*}$ :

$$
L_{i j}=\left\{\begin{array}{lll}
\delta_{i j}\left[\left(A s^{*}\right)_{i}-s^{*} A s^{*}\right] & \text { if } & s_{i}^{*}=0 \\
s_{i}^{*}\left[a_{i j}-\left(s^{*} A\right)_{j}-\left(A s^{*}\right)_{j}\right] & \text { if } & s_{i}^{*} \neq 0
\end{array}\right.
$$

Hence the coordinates of the column vector $[L z]_{3 \times 1}$ are

$$
(L z)_{k}=s_{k}^{*} \sum_{j=1}^{3}\left[a_{k j}-\left(s^{*} A\right)_{j}-\left(A s^{*}\right)_{j}\right] z_{j}, \quad k=1,2,3 .
$$

From $z \in \operatorname{Ker} Q$ we get

$$
C L z=s_{1}^{*} \sum_{j=1}^{3}\left[a_{1 j}-\left(s^{*} A\right)_{j}-\left(A s^{*}\right)_{j}\right] z_{j}=0 .
$$

Applying equation (12) and condition $s_{1}^{*} \neq 0 \quad\left(s^{*} \in \stackrel{\circ}{\Delta}^{3}\right)$ we have that

$$
\begin{aligned}
\sum_{j=1}^{3}\left[a_{1 j}-\left(s^{*} A\right)_{j}-\left(A s^{*}\right)_{j}\right] z_{j} & =\left[\left(-a_{12}+a_{13}\right)+\left(s^{*} A\right)_{2}+\left(A s^{*}\right)_{2}-\left(s^{*} A\right)_{3}-\left(A s^{*}\right)_{3}\right] z_{3} \\
& =\left[\left(-a_{12}+a_{13}\right)+\sum_{i} s_{i}^{*}\left(a_{i 2}+a_{i 3}\right)=0 .\right.
\end{aligned}
$$

From this by (10) we obtain $z_{3}=0$ and by (12) also $z=0$. Therefore from Theorem 2.3 we have that the system (11) is locally observable at $s^{*}$.

Remark 3.6. Of course, the above theorem can be immediately generalized to the case when the observation function correspond to another coordinate of the phenotypic frequencies vector, that is, $h(s)=s_{k}$, with $k=2$ or 3 .

Remark 3.7. The condition (10) of Theorem 3.5 reduces to $a_{12} \neq a_{13}$, if the play-off matrix $A$ is symmetric.

Next we present another result on observability in which the observed function are given by the addition of two of the coordinates of phenotypic state vector, for example, $h(s)=s_{1}+s_{2}$. Although the observation of $s_{1}+s_{2}$ 
is clearly equivalent to the observation of $s_{3}$, the theorem below will provide an alternative sufficient condition for local observability. To this end we introduce the following definition and notation.

Definition 3.8. Given $A \in \mathbb{R}^{n \times n}$, the matrix $\bar{A}=\left(\bar{a}_{i j}\right)$ will be called the symmetrized matrix of where

$$
\bar{a}_{i j}=\frac{a_{i j}+a_{j i}}{2} \quad i, j \in\{1,2, \ldots, n\} .
$$

Theorem 3.9. Given the pay-off matrix $A_{3 \times 3}$, let us suppose that the condition

$$
\frac{1}{2} \sum_{i=1}^{2}(-1)^{i} s_{i}^{*} a_{i \bullet}+\left(s_{1}^{*}-s_{2}^{*}\right) \sum_{j, k=1}^{3} s_{k}^{*} \bar{a}_{k j} \neq 0 \quad\left(\text { where } a_{i} \bullet=\sum_{j} a_{i j}\right)
$$

is verified.

Then the observation system

$$
\left\{\begin{aligned}
\dot{s}_{i} & =f(s)=s_{i}\left[(A s)_{i}-s A s\right] \\
y & =h(s)=s_{1}+s_{2}
\end{aligned}\right.
$$

is locally observable in $\stackrel{\circ}{\Delta}^{3}$ at the equilibrium $s^{*}$.

Proof. Let $z \in T_{*} \cap \operatorname{Ker} Q$, and let us denote $h(s)=s_{1}+s_{2}=I s$ where $I:=\left(\begin{array}{lll}1 & 1 & 0\end{array}\right)$. Then $C=h^{\prime}\left(s^{*}\right)=I$.

On the other hand, by $z \in \operatorname{Ker} Q$, we have

$$
C z=I z=z_{1}+z_{2}=0 \quad \Rightarrow \quad z_{2}=-z_{1},
$$

implying $z_{3}=0$, since $z \in T_{*}$.

According to (13), we have

$$
(L z)_{k}=s_{k}^{*} \sum_{j=1}^{3}\left[a_{k j}-\left(s^{*} A\right)_{j}-\left(A s^{*}\right)_{j}\right] z_{j}, \quad k=1,2,3
$$


implying

$$
\begin{aligned}
C L z & =\sum_{i=1}^{3} I_{i} s_{i}^{*} \sum_{j=1}^{3}\left[a_{i j}-\left(s^{*} A\right)_{j}-\left(A s^{*}\right)_{j}\right] z_{j} \\
& =s_{1}^{*} \sum_{j=1}^{3}\left[a_{1 j}-\left(s^{*} A\right)_{j}-\left(A s^{*}\right)_{j}\right] z_{1}+s_{2}^{*} \sum_{j=1}^{3}\left[a_{2 j}-\left(s^{*} A\right)_{j}-\left(A s^{*}\right)_{j}\right] z_{2} \\
& =\left(s_{1}^{*}\left[\sum_{j} a_{1 j}-\sum_{j}\left(s^{*} A\right)_{j}-\sum_{j}\left(A s^{*}\right)_{j}\right]-s_{2}^{*}\left[\sum_{j} a_{2 j}-\sum_{j}\left(s^{*} A\right)_{j}-\sum_{j}\left(A s^{*}\right) j\right]\right) z_{1} \\
& =\left[s_{1}^{*} \sum_{j} a_{1 j}-s_{2}^{*} \sum_{j} a_{2 j}+\sum_{j}\left(s^{*} A\right)_{j}\left(s_{2}^{*}-s_{1}^{*}\right)+\sum_{j}\left(A s^{*}\right)_{j}\left(s_{2}^{*}-s_{1}^{*}\right)\right] z_{1} \\
& =\left[s_{1}^{*} a_{1} \bullet s_{2}^{*} a_{2} \bullet\left(s_{2}^{*}-s_{1}^{*}\right) \sum_{j} \sum_{k} s_{k}^{*}\left(a_{k j}+a_{j k}\right] z_{1}\right. \\
& =2\left[\frac{1}{2}\left(s_{1}^{*} a_{1 \bullet}-s_{2}^{*} a_{2} \bullet\right)+\left(s_{2}^{*}-s_{1}^{*}\right) \sum_{j, k} s_{k}^{*} \frac{1}{2}\left(a_{k j}+a_{j k}\right)\right] z_{1} \\
& =-2\left[\frac{1}{2} \sum_{i=1}^{2}(-1)^{i} s_{i}^{*} a_{i} \bullet+\left(s_{1}^{*}-s_{2}^{*}\right) \sum_{j, k} s_{k}^{*} \bar{a}_{k j}\right] z_{1}=0 .
\end{aligned}
$$

Hence by (14), we get $z_{1}=0$ and by (16) $z_{2}=0$, implying $z=0$. Therefore, again by Theorem 2.3 system (15) is locally observable at the equilibrium $s^{*}$.

Remark 3.10. This theorem permits us to separate the frequencies of the phenotypes 1 and 2, observing their sum. The theorem can be adapted immediately, to the case in which the observed function is the sum of any two coordinates of phenotypic state vector.

\section{Examples for the observability}

We are going to see some concrete examples where we can apply the results of Theorems 3.5 and 3.9 .

Example 3.11. For a given $\epsilon \in \mathbb{R}^{+}$, we consider the replicator dynamics (7) with the following pay-off matrix

$$
A:=\left(\begin{array}{rrr}
-\epsilon & 1 & -1 \\
-1 & -\epsilon & 1 \\
1 & -1 & -\epsilon
\end{array}\right)
$$


The corresponding matrix game is a kind of "rock-scissors-paper" game, see Hofbauer and Sigmund (1988), also Kósa and Varga (1996). It is known that $S^{*}:=(1 / 3,1 / 3,1 / 3)$ is an ESS.

We assume now that we observe the phenotype frequency of the individuals of type 1 , that is, $h(s):=s_{1}$ with $s \in \Delta^{3}$. Since

$$
-a_{12}+a_{13}+\sum_{i=1}^{3} s_{i}^{*}\left[\left(a_{i 2}+a_{2 i}\right)-\left(a_{i 3}+a_{3 i}\right)\right]=-1-1+\frac{1}{3} \cdot 0=-2 \neq 0
$$

verifying condition (10) and by the Theorem 3.5 we get that the considered system is locally observable at the equilibrium $S^{*}=\left(\frac{1}{3}, \frac{1}{3}, \frac{1}{3}\right)$.

Example 3.12. We consider the replicator dynamics (7) with the following pay-off matrix

$$
A:=\left(\begin{array}{rrr}
0 & -1 & 3 \\
2 & 0 & -1 \\
-1 & 2 & 0
\end{array}\right)
$$

This is a classical rock-scissors-paper game, therefore applying a necessary and sufficient condition in this type of games for the calculation of ESS (see Hofbauer Sigmund) it is easy to check that $S^{*}=\frac{1}{26}(9,10,7)$ is an ESS for this matrix. Let us check the sufficient condition (14) of Theorem 3.9 in order to obtain the local observability of system (15) at $S^{*}$.

The symmetrized matrix of $A$ is

$$
\bar{A}:=\left(\begin{array}{ccc}
0 & \frac{1}{2} & 1 \\
\frac{1}{2} & 0 & \frac{1}{2} \\
1 & \frac{1}{2} & 0
\end{array}\right)
$$

on the other hand,

$$
a_{1} \bullet=\sum_{j} a_{1 j}=0+(-1)+3=2, \quad a_{2} \bullet=2+0-1=1 .
$$

Hence if we calculate (14), we obtain that

$$
\frac{1}{2} \sum_{i=1}^{2}(-1)^{i} s_{i}^{*} a_{i \bullet}+\left(s_{1}^{*}-s_{2}^{*}\right) \sum_{j, k=1}^{3} s_{k}^{*} \bar{a}_{k j}=\frac{-69}{338} \neq 0 .
$$


Therefore, applying the Theorem 3.9 we deduce that the considered system is locally observable at $S^{*}=\frac{1}{26}(9,10,7)$ (see Figure 1 )

\section{Observability of strategic model of selection.}

In this section, for the sexual populations, we shall deal with observation systems corresponding to different phenotypic characteristics. We suppose that in a large panmictic diploid population there are $n$ alleles $A_{1}, \ldots, A_{n}$ at an autosomal locus and the phenotype of a zygote is uniquely determined by its genotype: for each genotype $A_{i} A_{j}$, let $S_{i j} \in \Delta_{N}$ be the phenotype of a zygote $A_{i j}$ ( $\mathrm{N}$ is the number of pure phenotypes). An individual mixed phenotype $s \in \Delta_{N}$ has the usual probabilistic interpretation, $s_{k}$ is the probability for an individual to exhibit the pure phenotype $k$. We emphasize that $S_{i j}$ and $S_{j i}$ are not supposed to be the same.

For each vector of allelic state $p \in \Delta_{n}$, the vector

$$
S(p):=\sum_{i, j=1}^{n} p_{i} p_{j} S_{i j}
$$

is the mean phenotype of the population. On the other hand, given a pay-off matrix $A \in \mathbb{R}^{N \times N}$, the viability (or fitness) of a zygote $A_{i} A_{j}$ of a population in allelic state $p \in \Delta_{n}$ is defined as

$$
S_{i j} \cdot A S=\sum_{k, l=1}^{N} S_{i j, k} a_{k l} S_{l}(p)
$$

where $\cdot$ stands for the scalar product of vectors, $S_{i j, k}$ and $S_{l}(p)$ are the $k$-th and the $l$-th components of the vectors $S_{i j}$ and $S(p)$, respectively.

For each $p \in \Delta_{n}$, the vector

$$
\bar{S}^{i}(p):=\sum_{j=1}^{n} p_{j} \bar{S}_{i j} \quad(i \in\{1, \ldots, n\}
$$

is the effective (or marginal) phenotype of allele $A_{i}$, where $\bar{S}_{i j}=\frac{1}{2}\left(S_{i j}+S_{j i}\right)$. 
Figure 1: Observability of the replicator dynamics. Example 3.12. 
Let now $p(t)$ be the vector of allelic frequency in the adult population at time $t$. Then the continuous model-time is given by the following system

$$
\dot{p}_{i}=p_{i}\left[\bar{S}^{i}(p)-\bar{S}(p)\right] A \bar{S}(p) ; \quad i \in\{1, \ldots, n\}
$$

where $\bar{S}(p):=\sum_{k, l=1}^{n} p_{i} p_{j} \bar{S}_{i j}$. It is easy to check that $\bar{S}(p)=S(p)$ in terms of which the model (20) reads as follows,

$$
\dot{p}_{i}=p_{i}\left[\bar{S}^{i}(p)-S(p)\right] A S(p) ; \quad i \in\{1, \ldots, n\} .
$$

Throughout this section we shall suppose that $S^{*}$ is a polymorphic ESS $\left(S^{*} \in \stackrel{\circ}{\Delta}_{N}\right)$ and $p^{*}$ is a polymorphic allelic state $\left(p^{*} \in \stackrel{\circ}{\Delta}_{N}\right)$ realizing this ESS: $S\left(p^{*}\right)=S^{*}$. Moreover $p^{*}$ is an equilibrium of the dynamics (21) (see Cressman et al. (1996)).

Finally, we check that the interior $\stackrel{\circ}{\Delta}_{n}$ of simplex $\Delta_{n}$ is an $(n-1)$ dimensional regular sub-manifold of $\mathbb{R}^{n}$ positively invariant for the strategic dynamics and the simplex $\Delta_{n}$ is positively invariant for this dynamic too. For that uniquely it is enough to apply Theorems 3.2 and 3.4 considering dynamics (8) with

$$
g_{i}(x):=S^{i}(x) \cdot A S(x)
$$

Now we present some results giving sufficient conditions for local observability of the strategic model at the equilibrium $p^{*}$.

\section{Observation of mean phenotype and effective phenotype}

If we consider dynamics (21) with the observation function defined by

$$
h: \mathbb{R}^{n} \rightarrow \mathbb{R}^{N}, \quad h(p):=S(p)-S^{*}
$$

where $S$ now stands for the obvious extension of $S$ in (18), to $\mathbb{R}^{n}$. For the effective phenotypes at the equilibrium of the dynamics, we introduce the notation $\bar{S}^{* i}:=\bar{S}^{i}\left(p^{*}\right) \quad(i \in\{1, \ldots, n\})$. 
Theorem 4.1. If the vectors

$$
\bar{S}^{* 1}, \bar{S}^{* 2}, \ldots, \bar{S}^{* n}
$$

are linearly independent then observation system (21)-(22) is locally observable at equilibrium $p^{*}$.

Proof. In order to apply the Theorem 2.3, it is not even necessary to linearize of the dynamics, since we easily obtain the derivative of $h$ at $p^{*}$,

$$
C=h^{\prime}\left(p^{*}\right)=2\left[\bar{S}^{* 1}, \bar{S}^{* 2}, \ldots, \bar{S}^{* n}\right] .
$$

Let $z \in T_{*} \cap \operatorname{Ker} Q$, then $C z=0$ implying $\sum_{i=1}^{n} z_{i} \bar{S}^{* i}=0$. Hence, by the independence of vectors $\bar{S}^{* i}(i \in\{1, \ldots, n\})$, we have $z=0$ which implies the local observability of considered system at $p^{*}$ which was to be proved.

Remark 4.2. From Gámez (2003) we recall that the linear independence of vectors $\bar{S}^{* 1}, \ldots, \bar{S}^{* n}$, in cases of Mendelian symmetry, implies asymptotic stability of the equilibrium $p^{*}$.

Remark 4.3. Since the linear independence of vectors $\bar{S}^{* 1}, \ldots, \bar{S}^{* n}$ is equivalent to the geometric transversality condition (3) of Theorem 2.3, the unique way of applying the general sufficient condition of Theorem 2.3 is requiring the lineal independence of the effective phenotypes at the equilibrium $p^{*}$.

Let us suppose now that for some $i \in\{1, \ldots, n\}$ of $(21)$, we observe the effective phenotype of allele $A_{i}$, that is

$$
h: \mathbb{R}^{n} \rightarrow \mathbb{R}^{N}, \quad h(p):=\bar{S}^{i}(p)-\bar{S}^{i}\left(p^{*}\right)
$$

where $\bar{S}^{i}$ stands for the extension of $\bar{S}^{i}$ in (19), to $\mathbb{R}^{n}$.

Similarly to the proof of Theorem 4.1, we obtain the following sufficient condition for the observability of the considered model:

Theorem 4.4. If the vectors

$$
\bar{S}_{i 1}, \bar{S}_{i 2}, \ldots, \bar{S}_{i n}
$$

are linearly independent then the observation system (21)-(23) is locally observable at $p^{*}$. 


\section{Partial observation of the mean phenotype}

We consider again dynamics (21), however, with the observation defined by the following function

$$
h: \mathbb{R}^{n} \rightarrow \mathbb{R}, \quad h(p):=S_{1}(p)-S_{1}\left(p^{*}\right)
$$

where $S_{1}(p)$ is the first coordinate of mean phenotype $S(p)$.

For the case $N=3$, the following theorem provides sufficient conditions for the local observability at the equilibrium $p^{*}$, with an important difference in comparison to the previous theorems: In the present case items of the pay-off matrix are also involved in the sufficient conditions. In this sense the following theorem also illustrates how the considered dynamics can help to guarantee local observability without local invertibility of the observation function $h$.

Theorem 4.5. If

$$
\begin{aligned}
& \bar{S}_{1}^{* 1}=\bar{S}_{1}^{* 2}, \\
& \bar{S}_{1}^{* 1} \neq \bar{S}_{1}^{* 3}
\end{aligned}
$$

and

$$
\sum_{j=2}^{3} \sum_{i=1}^{3}\left(\bar{S}_{i}^{* 3}-S_{i}^{*}\right) a_{i j}\left(\bar{S}_{j}^{* 1}-\bar{S}_{j}^{* 2}\right) \neq 0
$$

then the observation system (21)-(24) is locally observable at the equilibrium allelic state $p^{*}$.

Proof. Let $z \in T_{*} \cap \operatorname{Ker} Q$. Then from

$$
C=h^{\prime}\left(p^{*}\right)=2\left[\bar{S}_{1}^{* 1}, \bar{S}_{1}^{* 2}, \bar{S}_{1}^{* 3}\right]
$$

we have $C z=\sum_{i=1}^{3} 2 \bar{S}_{1}^{* i} z_{i}$. Hence by (25) and $z \in T_{*} \cap \operatorname{Ker} Q$, we have $C z=2\left[\bar{S}_{1}^{* 1}\left(z_{1}+z_{2}\right)+\bar{S}_{1}^{* 3} z_{3}\right]=2\left[\bar{S}_{1}^{* 1}\left(-z_{3}\right)+\bar{S}_{1}^{* 3} z_{3}\right]=2\left(-\bar{S}_{1}^{* 1}+\bar{S}_{1}^{* 3}\right) z_{3}=0$

Therefore, by (26) we get $z_{3}=0$ and from $z \in T_{*}$, we obtain that $z_{1}=-z_{2}$. 
On the other hand, for the linearization of model we calculate the Jacobian of the right-hand side of the dynamics:

$$
L_{i j}=p_{i}^{*}\left[\left(\bar{s}_{i j}-2 \bar{S}^{* j}\right) A S^{*}+2\left(\bar{S}^{* i}-S^{*}\right) A \bar{S}^{* j}\right] \quad(i, j=1,2,3),
$$

for the coordinates of the vector $(L z)_{3 \times 1}$ we have

$$
(L z)_{k}=p_{k}^{*}\left(\bar{S}^{* k}-S^{*}\right) A 2 \sum_{i=1}^{3} \bar{S}^{* i} z_{i}, \quad(k=1,2,3) .
$$

Therefore,

$$
C L z=2 \sum_{j=1}^{3} \bar{S}_{1}^{* j} p_{j}^{*}\left(\bar{S}^{* j}-S^{*}\right) A 2 \sum_{i=1}^{3} \bar{S}^{* i} z_{i}=0 \quad(z \in \operatorname{Ker} Q) .
$$

If we develop $\sum_{j=1}^{3} \bar{S}_{1}^{* j} p_{j}^{*}\left(\bar{S}^{* j}-S^{*}\right)$, we obtain

$$
\sum_{j=1}^{3} \bar{S}_{1}^{* j} p_{j}^{*}\left(\bar{S}^{* j}-S^{*}\right)=\bar{S}_{1}^{* 1}\left[p_{1}^{*}\left(\bar{S}^{* 1}-S^{*}\right)+p_{2}^{*}\left(\bar{S}^{* 2}-S^{*}\right)\right]+\bar{S}_{1}^{* 3} p_{3}^{*}\left(\bar{S}^{* 3}-S^{*}\right)
$$

where

$$
\begin{aligned}
p_{1}^{*}\left(\bar{S}^{* 1}-S^{*}\right)+p_{2}^{*}\left(\bar{S}^{* 2}-S^{*}\right) & =p_{1}^{*} \bar{S}^{* 1}+p_{2}^{*} \bar{S}^{* 2}-\left(p_{1}^{*}+p_{2}^{*}\right) S^{*} \\
& =S^{*}-p_{3}^{*} \bar{S}^{* 3}-\left(1-p_{3}^{*}\right) S^{*}=-p_{3}^{*}\left(\bar{S}^{* 3}-S^{*}\right),
\end{aligned}
$$

that is,

$$
\sum_{j=1}^{3} \bar{S}_{1}^{* j} p_{j}^{*}\left(\bar{S}^{* j}-S^{*}\right)=\left(-\bar{S}_{1}^{* 1}+\bar{S}_{1}^{* 3}\right) p_{3}^{*}\left(\bar{S}^{* 3}-S^{*}\right)
$$

Now developing $\sum_{i=1}^{3} \bar{S}^{* i} z_{i}$ we obtain

$$
\sum_{i=1}^{3} \bar{S}^{* i} z_{i}=\bar{S}^{* 1} z_{1}+\bar{S}^{* 2} z_{2}=\left(\bar{S}^{* 1}-\bar{S}^{* 2}\right) z_{1}
$$

since $z_{3}=0$ and $z_{2}=-z_{1}$.

Substituting (29) and (30) into (28), we get

$$
C L z=2\left(-\bar{S}_{1}^{* 1}+\bar{S}_{1}^{* 3}\right) p_{3}^{*}\left(\bar{S}^{* 3}-S^{*}\right) A 2\left(\bar{S}^{* 1}-\bar{S}^{* 2}\right) z_{1} .
$$


From $p_{3}^{*}>0$ since $p^{*} \in \stackrel{\circ}{\Delta^{3}}$ we have $p_{3}^{*}>0$ and by hypothesis $-\bar{S}_{1}^{* 1}+$ $\bar{S}_{1}^{* 3} \neq 0$, implying

$$
\left(\bar{S}^{* 3}-S^{*}\right) A\left(\bar{S}^{* 1}-\bar{S}^{* 2}\right) z_{1}=0 .
$$

If we calculate now the vectors $\left(\bar{S}^{* 3}-S^{*}\right) A \in \mathbb{R}^{1 \times 3}$ and $\left(\bar{S}^{* 1}-\bar{S}^{* 2}\right) \in \mathbb{R}^{3 \times 1}$ by coordinates, we obtain

$$
\left[\left(\bar{S}^{* 3}-S^{*}\right) A\right]_{l}=\sum_{i=1}^{3}\left(\bar{S}_{i}^{* 3}-S_{i}^{*}\right) a_{i l}, \quad(l=1,2,3)
$$

and

$$
\left[\bar{S}^{* 1}-\bar{S}^{* 2}\right]_{k}=\bar{S}_{k}^{* 1}-\bar{S}_{k}^{* 2}, \quad(k=2,3) .
$$

where by $(25),\left[\bar{S}^{* 1}-\bar{S}^{* 2}\right]_{1}=0$. Hence,

$$
\left(\bar{S}^{* 3}-S^{*}\right) A\left(\bar{S}^{* 1}-\bar{S}^{* 2}\right)=\sum_{j=2}^{3} \sum_{i=1}^{3}\left(\bar{S}_{i}^{* 3}-S_{i}^{*}\right) a_{i j}\left(\bar{S}_{j}^{* 1}-\bar{S}_{j}^{* 2}\right),
$$

which by (27) is different from zero. The lather, together with (31) imply that $z_{1}=0$ and so $z=0$. Therefore, according to the Theorem 2.3 the system is locally observable at $p^{*}$, which complete the proof.

Remark 4.6. This theorem can be adapted in an obvious way to the case obtain by permutation of the corresponding indices.

\section{Illustration of Theorem 4.5.}

In the following we present an example (the so called case of semi-dominance) where we apply Theorem 4.5.

We assume that the homozygotes exhibit pure phenotypes represented by the canonical basic vectors of $\mathbb{R}^{3}$ :

$$
S_{11}:=e_{1}, \quad S_{22}:=e_{2}, \quad S_{33}:=e_{3} .
$$

Then the dominance structure is represented by the hypermatrix

$$
\left[S_{i j}\right]_{3 \times 3}=\left(\begin{array}{ccc}
e_{1} & \alpha_{12} e_{1}+\left(1-\alpha_{12}\right) e_{2} & \alpha_{13} e_{1}+\left(1-\alpha_{13}\right) e_{3} \\
\alpha_{21} e_{2}+\left(1-\alpha_{21}\right) e_{1} & e_{2} & \alpha_{23} e_{2}+\left(1-\alpha_{23}\right) e_{3} \\
\alpha_{31} e_{3}+\left(1-\alpha_{31}\right) e_{1} & \alpha_{32} e_{3}+\left(1-\alpha_{32}\right) e_{2} & e_{3}
\end{array}\right)
$$


with $\alpha_{i j} \in[0,1], \quad i, j \in\{1,2,3\}$.

Particular cases:

1. If $\alpha_{i j}=\alpha_{j i}=1 \quad(i, j=1,2,3)$ we have a paternal inheritance system.

2. If $\alpha_{i j}=\alpha_{j i}=0 \quad(i, j=1,2,3)$ we get a maternal inheritance system.

3. If $\alpha_{i j}=\alpha \quad(i, j=1,2,3)$ we obtain a convex combination of the paternal and maternal inheritance.

4. Suppuse $\alpha_{i j}=1-\alpha_{j i} \quad(i, j=1,2,3)$. Then the hipermatrix $\left[S_{i j}\right]$ is symmetrical. In order to study this case, we consider the dynamics (21) with the pay-off matrix $A$ of (17) with $\epsilon=1$. Now we know that $S^{*}:=\left(\frac{1}{3}, \frac{1}{3}, \frac{1}{3}\right)$ is an ESS. In order to simplify the calculations, we take $\alpha_{i j}=\frac{1}{2}$, for all $i, j$. From the corresponding calculations we get

$$
\bar{S}^{* 1}=\frac{1}{6}(4,1,1), \quad \bar{S}^{* 2}=\frac{1}{6}(1,4,1), \quad \bar{S}^{* 3}=\frac{1}{6}(1,1,4)
$$

implying

$$
\sum_{j=2}^{3} \sum_{i=1}^{3}\left(\bar{S}_{i}^{* 1}-S_{i}^{*}\right) a_{i j}\left(\bar{S}_{j}^{* 2}-\bar{S}_{j}^{* 3}\right)=\frac{1}{2} \neq 0 .
$$

Therefore by Theorem 4.5 we obtain that the considered system is locally observable at the equilibrium.

\section{Conclusion}

The approach of mathematical systems theory as an effective tool in discovering certain qualitative properties of dynamic evolutionary models.

Earlier results on local observability have been extended, on the one hand, to the replicator dynamics describing the phenotypic evolution of an asexual population. On the other hand, for sexual populations, we provided extensions to non-symmetric inheritance patterns such as the maternal (paternal) 
and the semi-dominant ones. Because of the nonlinearity of the models the observability results are only local, admitting to recover the state of system from the observed phenotypic characteristics near equilibrium.

\section{References}

Cressman, R.; Hofbauer, J.; Hines, W.G.S., 1996. Evolutionary stability in strategic models of single-locus frequency-dependent viability selection. Journal of Mathematical Biology.

Cressman, R.; Garay, J.; Varga, Z., 2003. Evolutionarily State set in the Single-locus Frequency-Dependent model of Natural Selection, Journal of Mathematical Biology, (in press).

Gámez, M.; Carreño, R.; Kósa, A.; Varga, Z., 2003. Observability in strategic models of viability selection, BioSystems (in press).

Garay, J. and Garay, M.B., 1998. Genetical reachability: When does a sexual population realize all phenotypic states. J. Math. Biol. 37, 146-154.

Garay, J.; Varga, Z., 1998. When will a sexual population evolve to an ESS?. Proc. R. Soc. Lond. B, 265, 1007-1010.

Hofbauer, J.; Sigmund, K., 1988. The Theory of Evolution and Dynamical Systems, 1988. Cambridge: Cambridge University Press.

Kalman, R.E.; Falb, P.L.; Arbib, M.A., 1969. Topics in Mathematical System Theory. McGraw-Hill, New York.

Kósa, A.; Varga, Z., 1996. Controllability of evolutionary game dynamics. Bull. Univ. Agric. Sci. Gödöllo (II), 75, 43-49.

Lee, E.B.; Markus, L., 1967. Foundations of Optimal Control Theory. Wiley, New York.

Maynard Smith, J. and Price, J., 1973. The logic of animal conflicts. Nature 249, 15-18.

Scarelli, A.; Varga, Z., 2002. Controllability of selection-mutation systems. BioSystems, 65, 113-121.

Taylor, P.D.; Jonker, L.B., 1978. Evolutionarily stable strategies and games dynamics. Math. Biosciences, 40, 145-156. 
Varga, Z., 1989. On controllability of Fisher's model of selection. In: Dafermos, C.M., Ladas, G., Papanicolaou, g. Proc. EQUADIFF Conference, 717-723.

Varga, Z., 1992. On observability of Fisher's model of selection. Pure Math. and Appl. Ser. B, 1, 15-25.

Varga, Z.; Scarelli, A.; Shamandy, A., 2003. State monitoring of apopulation system in changing environment (Community Ecology, in press). 